\title{
Efficiency of Voice Therapy for Persons with Vocal Tremors
}

\author{
Noorain Alam*, Sanjay Munjal and Naresh Panda \\ Department of ENT, Post Graduate Institute Medical Education \& Research, India
}

Submission: July 07, 2017; Published: July 17, 2017

*Corresponding author: Noorain Alam, Lecturer, Speech \& Hearing unit, ENT dept, Post Graduate Institute Medical Education \& Research, Chandigarh, India, mobile: +91-9033 747078; Email: noorain.aslp@gmail.com

\begin{abstract}
Introduction: Essential vocal tremor (EVT) is vocal tremor occurring in an individual diagnosed with essential tremor and is an action induced tremor that occurs during speech production. It causes fewer communication attempts, difficulty initiating and maintaining phonation, effortful speaking avoidance of social events trouble sustaining relationship leading to impair quality of life for such persons.

Aim of the study: The aim of present study was to find out the efficacy of voice therapy in persons with voice tremors which may be shown in improvement in acoustic analysis and quality of life measures.

Methodology: The study was conducted at ENT department of PGIMER, Chandigarh. Two subjects were included in the study having a complaint of vocal tremors. A 65-year-old male patient and a 68 -year old female patient both diagnosed with EVT. The acoustic assessment was done using Dr. Speech software. Effect of voice disorder on quality of life was assessed using Voice Handicap Index. Measurements were repeated after completion of a voice therapy program with a frequency of twice a week for eight weeks. A combination of Laryngeal maneuvers, Relaxation exercises and Breathing exercises were used in voice therapy protocol.

Results and Discussion: Pre-therapy and post-therapy comparison shows improvement in the quality of life shown by reduction of VHI scores across three parameters (functional, physical and emotional) of VHI in both the patients. Acoustic parameters of voice also showed improvement in terms of shimmer and HNR in both the patients.
\end{abstract}

Conclusion: The voice therapy shows improvement in the quality of life as well as acoustic parameters of voice in this population. More research is required in this area to find out the efficacy of this therapy protocol on a larger population.

Keywords: Essential Voice Tremors; Acoustic measurements; Quality of life; Voice Handicap Index Essential vocal tremor (EVT)

\section{Introduction}

\section{Efficiency of Voice Therapy for persons with vocal tremors}

Essential vocal tremor (EVT) is vocal tremor occurring in an individual diagnosed with essential tremor and is considered to be an action induced tremor that occurs during speech production [1,2]. EVT affects the aging populations most as it is neurogenic in origin, [3] and may be present in isolation or in association with other disorders such as with Parkinson's disease, amyotrophic lateral sclerosis, spinal muscular dystrophy, spasmodic dysphonia, and other types of essential tremor, such as those affecting the limbs or face [4]. Vocal tremors are present in musculature isolated to or combined with the larynx [5], tongue [6], jaw [6], face [7], head [7], and respiratory system [1]. Main symptoms of vocal tremors include rhythmic modulations of pitch and loudness causing a fluctuating in the amplitude and fundamental frequency of the voice. Vocal tremor modulation rates may range from $3-12 \mathrm{~Hz}$ during sustained phonation [8]. It causes fewer communication attempts, difficulty initiating and maintaining phonation, effortful speaking avoidance of social events trouble sustaining relationship leading to impair quality of life for such persons [9].

Vocal tremors result from a lesion in the extrapyramidal system or cerebellum, basal ganglia or thalamus [10]. Damage in these areas may disturb motor functions and coordination of muscles involving at any level of the speech production mechanism. Managing options for vocal tremors include Pharmacological drugs [11], Alcohol [11], Botox [12], and Deep brain stimulation [13]. Use of Voice Therapy - In literature, it has been reported that voice therapy techniques are generally ineffective in treating vocal tremor $[4,14]$. The need of study- An 


\section{Global Journal of Otolaryngology}

effective technique of voice therapy is the required which can reduce tremors in patients with vocal tremors as well as may be effective in improving the quality of life in such patients.

\section{Aim of the study}

The aim of present study was to find out the efficacy of voice therapy in persons with voice tremors which may be shown in improvement in acoustic analysis and quality of life measures.

\section{Methodology}

The study was conducted at ENT department of PGIMER, Chandigarh which is a premier tertiary healthcare center in India.

\section{Two subjects were included in the study having a complaint of vocal tremors.}

a. Case 1: A 65-year-old male patient was referred from Neurology department with a complaint of EVT to the speech and hearing unit, PGIMER, Chandigarh with a complaint of tremors in voice. Tremors were seen more during initiation of the conversation and led to breathlessness sometimes. Tremors were present in the right hand while writing. Perceptual voice assessment was done, which showed that Pitch and loudness variations were affected.

b. Case 2: A 68-year-old female patient was referred from Neurology department with a complaint of EVT to speech and hearing unit PGIMER, Chandigarh with a complaint of tremors in voice. The voice quality was strained. Tremors were seen more during initiation of the conversation and led to breathlessness sometimes. On perceptual voice assessment pitch and loudness, variations were affected.

Both cases underwent perceptual, acoustic assessments. The acoustic assessment was done using Dr. Speech software. Effect of voice disorder on quality of life was assessed using Voice Handicap Index Jacobson et al. [15]. Both patients were enrolled for voice therapy program with a frequency of twice a week for eight weeks.

A combination of Laryngeal maneuvers, Relaxation exercises (to reduce muscle tension in and around the head and neck region) and Breathing exercises were used.

Laryngeal lowering: Dromey et al. [16] found that lowpitch phonation reduced the amplitude rate of vocal tremor. They concluded that a lowered larynx may have contributed to relaxation and stability in the vocal system, resulting in the reduction of tremor severity. Laryngeal lowering was achieved using digital manipulation.

Yawn-sigh [17]: In this technique, the larynx drops to a low position, the tongue is more forward, there is slight opening between the vocal folds, and pharynx is usually dilated. The patient is asked to perform a yawn followed by relaxed sigh which is combined with phonation.
Easy voice onset: It facilitates reduced effort during voicing onset and to promote increased airflow through the vocal folds to achieve a slightly breathier and softer voice quality than typically exhibited [17].

Anterior focus: It was used to improve the use of vocal tract resonance for voice projection. At first, whisper is used to repeat front of the mouth words followed by repetition of light voice [17].

Differential relaxation exercise: It involves sequentially increasing tension in each body part and then relaxing it starting with either the feet and working upward to the head and neck and vice versa [17].

Breathing exercise: Laryngeal lowering/vocal tract lengthening also can be achieved through increases in lung volume, which cause the diaphragm and the supporting respiratory musculature to stabilize and lower the larynx through a diaphragmatic tracheal pull Iwarsson \& Sundberg [18] Iwarsson et al. [19]. These exercises include the use of forceful inhalation and exhalation and abdominal breathing techniques. Acoustical analyses of voice, as well as VHI, were re-administered after completion of voice therapy.

\section{Results}

Table 1: Acoustical Analysis in case 1 pre \& post therapy.

\begin{tabular}{|c|c|c|}
\hline $\begin{array}{c}\text { Acoustical } \\
\text { Parameters }\end{array}$ & Pre-Therapy & Post-therapy \\
\hline F0 Mean & 147.46 & 158.57 \\
\hline Jitter (\%) & 0.22 & 0.82 \\
\hline Shimmer (\%) & 5.02 & 2.14 \\
\hline Fo Tremor & 5.08 & 5.66 \\
\hline Mean Fo(Hz) & 147.46 & 158.57 \\
\hline HNR(db) & 24.00 & 25.18 \\
\hline
\end{tabular}

Pre and post therapy comparison for Acoustical analysis as well as VHI measures are given in the Tables 1-4. In Case 1 acoustical analysis Table 1 shows improvement in terms of shimmer and HNR. There was a slight increase in the jitter $(0.22$ to 0.82$)$. However, the value is within normal limits $(\leq 1.04 \%)$. While on VHI (Table 3) there is improvement across all three parameters i.e. functional, physiological and emotional. In Case 2 there was an improvement in all three parameters of the acoustic measures i.e. jitter, shimmer and HNR (Table 2). VHI shows improvement in all three parameters (Table 4).

Table 2: Acoustical Analysis in case 2 pre \& post therapy.

\begin{tabular}{|c|c|c|}
\hline $\begin{array}{c}\text { Acoustical } \\
\text { Parameters }\end{array}$ & Pre- & Post-therapy \\
\hline Therapy & Post-therapy & 158.57 \\
\hline F0 Mean & 211 & 267 \\
\hline Jitter (\%) & 2.75 & 1.82 \\
\hline Shimmer (\%) & 6.42 & 4.14 \\
\hline Fo Tremor & 2.78 & 1.09 \\
\hline HNR(dB) & 21.34 & 22.26 \\
\hline
\end{tabular}




\section{Global Journal of Otolaryngology}

Table 3: Voice Handicapped Index in case 1 pre \& post therapy.

\begin{tabular}{|c|c|c|}
\hline Parameters & Pre- & Post-therapy \\
\hline Therapy & Post-therapy & 158.57 \\
\hline Functional & 17 & 10 \\
\hline Physical & 24 & 19 \\
\hline Emotional & 11 & 4 \\
\hline Total score & 52 & 33 \\
\hline
\end{tabular}

Table 4: VHI shows improvement in all three parameters.

\begin{tabular}{|c|c|c|}
\hline Parameters & Pre- & Post-therapy \\
\hline Therapy & Post-therapy & 158.57 \\
\hline Functional & 18 & 14 \\
\hline Physical & 23 & 19 \\
\hline Emotional & 36 & 22 \\
\hline Total score & 77 & 55 \\
\hline
\end{tabular}

\section{Discussion}

Previous research has indicated that individuals with neurologically-based voice disorders, including EVT, often experience reduced quality of life [20]. Reduced quality of life may be related to fewer communication attempts, difficulty initiating and maintaining phonation, effortful speaking avoidance of social events trouble sustaining relationships [9]. In this study also persons with vocal tremors reported reduced quality of life as shown by a high score on VHI in both the subjects.

Pre-therapy and post-therapy comparison of VHI shows reduction in scores in all three parameters i.e. functional, physical and emotional in both the patients indicating an improvement of the quality of life in both the patients. Pre and post therapy comparison of acoustical analysis of voice also showed improvement in shimmer and HNR measures in both the patients. In case 1 there is a slight increase in jitter post therapy (from 0.22 to 0.82 ). However, the value is within normal limits only. Thus a combination of relaxation exercises, laryngeal lowering, and breathing exercise are effective in improvement in acoustical parameters as well as an increase in quality of life in persons with vocal tremors.

\section{Conclusion}

The study highlighted the efficacy of voice therapy in persons with vocal tremors. The voice therapy shows improvement in the quality of life as well as acoustic parameters of voice in this population. More research is required in this area to find out the efficacy of this therapy protocol on a larger population.

\section{References}

1. Hechinski VC, Thomsen IV, Buch NH (1975) The nature of primary vocal tremor. Can J Neurol Sci 2(3):195-197.
2. Warrick P, Dromey C, Irish J, Durkin L (2000) The treatment of essential voice tremor with botulinum toxin A: a longitudinal case report. J Voice 14(3): 410-421.

3. Finnegan EM, Luschei ES, Barkmeier JM, Hoffman, et al. (2003) Synchrony of laryngeal muscle activity in persons with vocal tremor. Archives of Otolaryngology- Head and Neck Surgery 129(3): 313-318.

4. Barkmeier-Kraemer J, Lato A, Wiley K (2011) Development of a speech treatment program for a client with essential vocal tremor. Semin Speech Lang 32(1): 43-57.

5. Koda J, Ludlow CL (1992) An evaluation of laryngeal muscle activation in patients with voice tremor. Otolangol Head Neck Surg 107(5): 684696.

6. Tallon-Barranco A, Vazquez A, Javier Jimenez Jimenez F, et al. (1997) Clinical features of essential tremor seen in neurology practice: a study of 357 patients. Parkinsonism Relat Disord 3(4): 187-190.

7. Lou JS, Jankovic J (1991) Essential tremor: clinical correlates in 350 patients. Neurology 41(2 Pt 1): 234-238.

8. Gamboa J, Jimenez-Jimenez FJ, Nieto A, et al. (1998) Acoustic voice analysis in patients with essential tremor. J Voice 12(4): 444-452.

9. Cohen SM, Dupont WD, Courey MS (2006) Quality-of-life impact of non neoplastic voice disorders: A meta-analysis. Ann Otol Rhinol Laryngol 115(2): 128-134.

10. Deuschl G, Raethjen J, Lindemann M, Krack P (2001) the pathophysiology of tremor. Muscle Nerve 24: 716-735.

11. Chen JJ, Swope DM (2007) Essential tremor. Journal of Pharmacy Practice 20(6): 458-468.

12. Sulica L, Louis ED (2010) Clinical characteristics of essential voice tremor: A study of 34 cases. Laryngoscope 120(3): 516-528.

13. Putzer M, Barry WJ, Moringlane JR (2008) Effect of bilateral stimulation of the sub thalamic nucleus on different speech subsystems in patients with Parkinson's disease. Clinical Linguistics and Phonetics 22(12): 957-973.

14. Boone DR, McFarlane SC (1993) A critical view of the yawn-sigh as a voice therapy technique. Journal of Voice 7(1): 75-80.

15. Jacobson BH, Johnson A, Grywalski C, Silbergleit A (1997) The Voice Handicap Index (VHI) development and validation, American Journal of Speech-Language Pathology 6: 66-70.

16. Dromey C, Warrick P, Irish J (2002) The influence of pitch and loudness changes on the acoustics of vocal tremor. J Speech Lang and Hear Res 45(5): 879-890.

17. Boon DR, McFarlane SC (1994) the Voice and Voice Therapy. ( $5^{\text {th }}$ edn). Mahwah, NJ: Prentice Hall

18. Iwarsson J, Sundberg J (1998) Effects of lung volume on vertical larynx position during phonation. Journal of Voice 12(2) 159-165.

19. Iwarsson J, Thomasson M, Sundberg J (1998) Effects of lung volume on the glottal voice source. J Voice 12(12): 424-433.

20. Cohen SM, Dupont WD, Courey MS (2006) Quality-of-life impact of nonneoplastic voice disorders: A meta-analysis. Annals of Otology, Rhinology and Laryngology 115(2) 128-134. 
This work is licensed under Creative Commons Attribution 4.0 License DOI: 10.19080/GJ0.2017.09.555751

\section{Your next submission with Juniper Publishers will reach you the below assets}

- Quality Editorial service

- Swift Peer Review

- Reprints availability

- E-prints Service

- Manuscript Podcast for convenient understanding

- Global attainment for your research

- Manuscript accessibility in different formats

( Pdf, E-pub, Full Text, Audio)

- Unceasing customer service

Track the below URL for one-step submission https://juniperpublishers.com/online-submission.php 